\title{
EDUCACIÓN AMBIENTAL: DE LA ACCIÓN A LA INVESTIGACIÓN
}

\author{
MAYER, M. \\ Centro Europeo dell'Educazione. Ministero Pubblica Istruzione. Frascati. Roma. Italia.
}

\begin{abstract}
SUMMARY
Environmental education has developed greatly over the last twenty years, reflecting upon the deep contradiction between the need for human development, which means the need for scientific development and a global market economy, and the need for a better quality of life and natural environment. In the educational proposals, the action for the environment is no more separated from a deeper understanding of the way we think and from the importance given to the emotional links with our planet. This wiser way to conceive environmental education is strongly concerned with the complexity of thought and the «reductionism» implicit in many pedagogical theories and environmental claims. Environmental education could become a new way to look at education, putting together the complexity theories, the importance of making sense of ignorance and uncertainty, and the need of constructing and comparing values. This new way to conceive education claims new educational structures and consistent strategies to use in research and evaluation: the action carried out all over the world in the last years indicates the issues and the strategies environmental education research has to face.
\end{abstract}

Caminante, son tus huellas el camino, y nada más; caminante, no hay camino, se hace camino al andar.

Antonio Machado

\section{INTRODUCCIÓN}

En todos los documentos internacionales, y también para la Unión Europea, la educación ambiental no se considera como una disciplina escolar autónoma sino como una enseñanza lo más interdisciplinar y transversal posible (Giolitto et al., 1997). Sin embargo, en los últimos diez años, educadores de todas las disciplinas, aunque en mayor medida enseñantes de disciplinas científicas, han decidido dedicar al medio ambiente y a la educación ambiental una parte de su labor didáctica.

¿Por qué? ¿A qué necesidades responde la educación ambiental? ¿Qué le hace ser diversa de la educación científica o de la educación cívica?

En este artículo quisiera hablar sobre las motivaciones que han impulsado a muchos educadores en Europa a ocuparse de educación ambiental, y también la manera en que la educación ambiental ha sido generalmente entendida y practicada, para poder después analizar el camino que se ha ido trazando en estos últimos años -las huellas que hemos dejado- y examinar algunas de las cuestiones que la educación ambiental propone. La educación ambiental, de hecho, parece haber trazado un camino similar y, sin embargo, de alguna manera crítico, respecto a la trayectoria de la educación científica, y resulta, por tanto, interesante pararse a reflexionar sobre el significado de lo que ya se ha hecho y sobre los problemas que se han puesto en evidencia.

Las reflexiones que siguen se basan en la experiencia desarrollada en el ámbito internacional a través del proyecto ENSI, de la OECD -en el cual he sido coordinadora nacional como representante del CEDE, Centro Europeo dell'Educazione, Centro de Investigación Educativa del Ministerio de Educación Italiano-, y de la experiencia de los últimos cuatro años como responsable, junto con mi colega Bruno Losito, del proyecto ORMEA relativo al «Observatorio sobre Investigación 
y Metodologías para la Educación Ambiental», relacionado con la puesta en marcha experimental en Italia de un sistema nacional para la educación ambiental. Las partes relativas a la complejidad y a su importancia en la educación ambiental están inspiradas en el módulo escrito para el master en educación ambiental propuesto por la Universidad Nacional de Educación a Distancia de Madrid (Mayer, 1994).

\section{IMÁGENES DE LA CIENCIA, DE LA ES- CUELA, DE LA SOCIEDAD}

Cuando en la década de los setenta -hace más de veinte años- se empezaba a hablar de educación ecológica, y después de educación ambiental, comenzaban a aflorar algunos de los elementos que han contribuido a modificar profundamente las imágenes de la ciencia, de la escuela y de la sociedad que se habían construido en la primera mitad de nuestro siglo y cuyas raíces se basaban en el éxito que el mito del progreso había recogido en el siglo precedente. Los jóvenes de los años setenta empezaban a darse cuenta de que: $a$ ) la ciencia y la tecnología no podían ser consideradas la solución a todos los problemas y que, por el contrario, eran causantes de problemas; $b$ ) el mito de la ilustración, según el cual el conocimiento es por sí mismo portador de valores democráticos y de mejor calidad de vida, era precisamente un mito; c) no era fácil definir qué es en realidad el progreso; d) la escuela, más que un instrumento de promoción social, era un instrumento de selección y de perpetuación del poder.

A una distancia de más de veinte años -con un debate todavía abierto sobre la complejidad y sobre la postmodernidad-, es interesante observar cómo todos estos elementos se han profundizado y difundido, pero también cómo todavía hoy en día las contradicciones que conllevan se nieguen cotidianamente:

- Se sigue sosteniendo un progreso científico y tecnológico basado en el uso indiscriminado de los recursos y, a la vez, se denuncia la gravedad de la situación medioambiental.

- Se afirma que educación y escuela ocupan el primer lugar en los intereses de cada nación y, sin embargo, casi siempre están en último lugar dentro de los compromisos presupuestarios.

- Se afirman los valores de la democracia, del respeto a la diversidad, de la solidaridad, cuando en todo el mundo y también en Europa, asistimos a una falta de tolerancia hacia los que son diferentes, cercanos o lejanos.

Los intereses, sobre todo comerciales tendentes a la «aldea global», y también los ideales económicos que miran hacia la unidad en Europa son, de hecho, sólo una cara de la moneda, mientras que la otra cara se compone de tendencias separatistas, de reivindicaciones locales, de movimientos religiosos, que fragmentan cada vez más esta unidad.
Ya no es fácil reconocer donde está el «progreso», donde está la «razón» y es, sin embargo, cada vez más importante aprender a rechazar las posturas extremas, a mediar entre homogeneidad y diversidad, entre uso y conservación de los recursos, entre libertad individual y necesidades colectivas.

Incluso dentro de la propia escuela, las contradicciones se hacen cada vez más evidentes: la escuela parece haber renunciado a su papel de depósito-biblioteca del conocimiento inmutable que se aprende de memoria, pero no está claro aún cuál será su nuevo papel. En todos los programas escolares, y no sólo en Europa, se habla de constructivismo y de aprendizaje activo y, a pesar de ello, disciplinas y contenidos siguen siendo más o menos los mismos (si acaso aumentan en extensión), las estructuras se modifican sólo superficialmente, a los maestros se les prepara casi igual que hace veinte años. La escuela realmente se ha dejado abandonada a su suerte, y las reformas poco pueden hacer si no se acompañan de un cambio más profundo, si no son los propios enseñantes, y los investigadores los que construyan juntos un camino más adecuado a las imágenes del mundo y de la ciencia que el nuevo siglo que viene trae consigo.

Ni tampoco en lo que se refiere al medio ambiente y a la educación ambiental faltan las contradicciones: se habla de desarrollo, y de desarrollo sostenible, y no queda claro dónde se halla la línea que separa desarrollo y sostenibilidad: en un documento oficial de la UNESCO (1992) realizado después del encuentro de Río, las palabras clave son: competitividad, educación y productividad, capital humano. Como señala Lucie Sauvé (1993), lo que todavía predomina, al menos en los documentos oficiales, es un paradigma sociocultural de tipo «industrial» y los paradigmas educativos correspondientes -el «racional»y el «tecnológico»-en que la educación se considera como una inversión económica fundamental para el desarrollo de la creatividad, de la productividad $y$ de la competitividad (UNESCO, 1992). El mismo capítulo 36 del programa 21 parece dar más importancia a la extensión de la información y, por tanto, a la cantidad, mientras que, contemporáneamente, el Foro Global de las ONG ponía énfasis (1993) en la calidad de la educación, en el carácter de construcción social del conocimiento, en el concepto de equidad como contraposición a un más superficial concepto de igualdad (Novo, 1995). En los documentos del Foro Global, el rol de la educación ya no es el de transmitir un saber determinado, la educación sí es considerada como un instrumento de «desarrollo», pero para la construcción de sociedades sostenibles, «fundadas en el respeto hacia todas las formas de vida», en las cuales se cuestionan los modelos socioeconómicos y se abre un espacio para «producir un nuevo saber, conjuntamente y a través de un enfoque crítico» (Sauvé, 1993).

Hasta ahora la educación ambiental ha utilizado las contradicciones para crecer y ha ofrecido una ocasión privilegiada de innovación educativa y de investigación para todos aquéllos que intentaban adecuar la escuela a los cambios profundos que se estaban produciendo en el mundo. Volver a recorrer el camino desarrollado por la 
educación ambiental en estos años, es por tanto, útil también para reflexionar sobre los errores cometidos, sobre los obstáculos todavía presentes, sobre los problemas por afrontar, no sólo por parte de la educación ambiental sino por toda la educación científica.

\section{LA EDUCACIÓN AMBIENTAL HACIA EL AÑO 2000}

Desde que se introdujo en la escuela y en la sociedad, la educación ambiental se ha ido modificando profundamente y se ha hecho cada vez más consciente de los profundos cambios que una nueva ética ambiental requiere no sólo en nuestros comportamientos, sino en nuestra concepción del conocimiento y del mundo. Si recorremos de nuevo la historia de la educación ambiental, vemos que desde las primeras «investigaciones del ambiente» de la escuela activa de los años cincuenta y sesenta, en las que el medio ambiente se veía como un expediente pedagógico que permitía implicar activamente a los alumnos, se pasa en los años setenta al reconocimiento de la importancia de la ecología, digna de ser enseñada junto con las otras «ciencias naturales» y, más tarde, con el descubrimiento del «riesgo» ambiental a finales de los setenta, a la introducción en los libros escolares de «nociones» sobre la importancia de los recursos naturales y sobre los posibles perjuicios de la contaminación.

La premisa implícita de esta primera forma de educación sobre el medio ambiente es que los problemas ambientales están causados por una falta de «conocimientos» y que la solución, por tanto, está en la información: Si la gente supiera, no se comportaría así. Aunque el problema es mucho más complejo -conocer los riesgos no es suficiente y, de hecho, no por casualidad, los países más contaminados son aquéllos en los que no sólo ciencia y tecnología están más desarrollados sino también el sistema educativo-, esta visión de la educación ambiental es todavía la que figura en la mayoría de los libros de texto, y todavía hoy son muchos los que parecen creer en el poder taumatúrgico de la «información objetiva».

Las razones por las que una «información correcta» no es suficiente -aunque no se niegue su utilidad-son de dos tipos:

1) La primera se refiere a la noción misma de información y de información objetiva: justamente en el debate sobre la ciencia moderna se pone en tela de juicio la posibilidad de que exista un conocimiento objetivo en sí mismo, por encima de visiones subjetivas, que represente fielmente el mundo real. En el campo ambiental, además, es fácil darse cuenta de que el conocimiento no es el único criterio para tomar decisiones: aunque es cierto que el riesgo, sobre el papel, de que se repitan accidentes como el de Chernobyl es extremadamente bajo - y será todavía menor cuando se vayan poco a poco cerrando las viejas centrales nucleares de Europa del Este-, ¿quién puede decidir que un riesgo tan improbable y al mismo tiempo tan grave es aceptable? ¿Quién está decidiendo que el riesgo del agujero de ozono -y del aumento, por tanto, de los casos de melanoma- no es suficiente para provocar la reconversión de la industria de los frigoríficos y de los acondicionadores de aire? ¿Quién decide que el riesgo del efecto invernadero no es lo suficientemente serio para dar lugar a medidas drásticas de control de la contaminación?

Con frecuencia los que tienen más competencias son justamente aquéllos que más pueden verse afectados por decisiones drásticas en materia de medio ambiente: técnicos y científicos al servicio de la industria o de intereses gubernamentales. El problema no es, por tanto, garantizar la «objetividad» de la información, sino la pluralidad de la información: hacer que se escuchen otros puntos de vista, permitir a todos que decidan autónomamente qué datos parecen más relevantes, qué riesgos correr, qué comportamientos cambiar.

2) La segunda razón por la cual la información no es suficiente tiene un carácter más estrictamente pedagógico. ¿Por qué razón la gente cambia sus comportamientos? El miedo no basta y el catastrofismo no paga. Hacen falta esperanzas y lazos estrechos con el medio ambiente que se pretende conservar. Un estudio realizado en Suecia -dentro del proyecto ENSI, y confirmado por otros estudios- ha puesto sobre la mesa que los grupos de alumnos donde se había dado más información sobre los riesgos ambientales y los problemas del planeta resultaban ser aquéllos en que los estudiantes se sentían más desconfiados, sin esperanza, incapaces de pensar posibles acciones para el futuro. Y, de hecho, los problemas ambientales se presentan hoy en día en su dimensión planetaria, tanto que nadie individualmente puede pensar poder hacer algo para resolverlos.

De estas consideraciones nacen y se desarrollan en los años ochenta las propuestas de educación en el ambiente. La educación en el ambiente reconoce que los comportamiento vienen guiados mucho más por nuestras emociones y valores que por los conocimientos y que, por tanto, es necesario no sólo ofrecer informaciones sino proponer experiencias que reconstruyan la conexión entre el hombre y el medio ambiente que se pretende conservar. Stephen Jay Gould, paleontólogo muy conocido por sus ensayos divulgativos y autor de libros $^{1}$ que ofrecen ejemplos de conocimiento científico más allá de los estereotipos afirma (1994):

«[...] me doy cuenta también de que no podemos vencer esta batalla para salvar especies y ambientes sin crear un vínculo emocional entre nosotros y la naturaleza, pues no lucharemos por salvar aquello que no amamos (sino que apreciamos sólo de un modo abstracto) [...] debemos hacer hueco a la naturaleza en nuestro corazón» (p. 40).

En este caso, la teoría implícita es que el vínculo emotivo con la naturaleza, el sentimiento que se intenta suscitar será lo suficientemente fuerte como para impulsar a un cambio en los comportamientos. Pero la educación en el ambiente ya ha dejado ver sus límites, no sólo porque la implicación es un proceso lento y fatigoso, que frecuentemente no consigue superar la edad de la adolescencia, 
-no sólo porque se da por supuesto que existen comportamientos «correctos» cuando en realidad la complejidad del problema hace que cambien rápidamente las propuestas de soluciones-, sino porque el concepto de medio ambiente que conlleva -entendido sobre todo como ambiente natural por conservar y proteger-contribuye a crear una nueva esquizofrenia: protegemos el ambiente «natural» y degradamos cada vez más el ambiente en el que vivimos.

En vez de integrar emociones y conocimientos, con frecuencia la educación en el ambiente descuida o empaña la claridad cognoscitiva: invoca a la protección de una naturaleza que ha demostrado en millones de años de saber valerse muy bien sin el hombre, y que seguramente sobrevivirá a nuestra especie. En realidad, lo que debemos proteger es la especie humana, una especie que se ha desarrollado en este planeta y en este ambiente natural; la destrucción del ambiente en el que hemos encontrado las condiciones favorables para nuestro desarrollo dejará espacio seguramente a otros equilibrios y otras formas de vida, como la extinción de los dinosaurios ha dado paso a los mamíferos y al hombre, pero puede ser extremadamente peligrosa precisamente para la supervivencia de nuestra especie.

El problema de la educación ambiental de los noventa es, por tanto, el de englobarlo todo - de las emociones a los conocimientos, de los valores a los comportamientossin presuponer relaciones de causa-efecto sino aceptando relaciones circulares, en las que los valores, emociones, conocimientos y comportamientos se refuerzan unos a otros. Desde este punto de vista de la educación ambiental, el medio ambiente ya no es necesariamente el natural, preservado, por conservar, y tampoco el tan lejano sobre el que el individuo no puede intervenir. El medio ambiente es el ambiente cercano, cotidiano, aquél en el que pequeñas iniciativas pueden comenzar a modificar actitudes, modos de actuar y formas de juzgar. Esta educación para el ambiente traduce en el campo educativo el lema «actuar localmente y pensar globalmente», y reconoce a la escuela un papel protagonista: no sólo de transmisión de informaciones, no sólo de uso más o menos pasivo de «propuestas verdes», sino de proposición de pequeñas acciones que partan del territorio y que incidan sobre el territorio constituyendo, como propone uno de los documentos elaborados por la Unión Europea (1994), un modelo de gestión del propio ambiente.

La educación para el ambiente también puede caer sin embargo en una trampa, la del activismo como un fin en sí mismo, el de la investigación de los resultados y de las soluciones, olvidando que las iniciativas en las escuelas son sólo un medio, un instrumento, para construir algo más: una conciencia ecológica que está hecha de conocimientos y emociones y no sólo de acciones, de reflexiones sobre las prácticas y los valores y no sólo de ecologismo militante.

Durante estos veinticinco años, por tanto, el carácter de la educación ambiental se ha ido modificando profundamente, no sólo porque los tres modos de hacer educación ambiental propuestos por Lukas (1980-81) -sobre, en y para- se han ido divulgando y poco a poco se han sustituido unos por otros, sino porque a menudo en este proceso se ha experimentado una reflexión, una evolución que ha permitido sacar a la luz las contradicciones y superarlas, para llegar a las actuales propuestas de muchas escuelas y de muchas asociaciones ecologistas que aceptan y proponen lo que se ha dado en llamar a veces un triple enfoque, y que podríamos denominar como enfoque complejo de la educación ambiental.

La educación ambiental ha demostrado hasta el momento ser flexible y capaz de generar propuestas adecuadas a un mundo en rápida evolución. No es tan claro, sin embargo, en qué medida esta flexibilidad depende del hecho de ser un ámbito que todavía se está conformando como disciplina -podríamos decir con Kuhn que está aún en una fase preparadigmática- o en qué medida se debe a la naturaleza misma de la educación ambiental. Una reflexión sobre el significado de las propuestas que parten de la educación ambiental permite, por tanto, no sólo conformar las características de las iniciativas de educación ambiental de calidad, sino también profundizar en la dimensión del cambio que la educación ambiental propone.

\section{CONOCER EL CONOCIMIENTO}

La integración de valores ambientales y valores educativos conlleva un verdadero desafío no sólo para la educación ambiental sino para el modo de concebir la educación (Losito y Mayer, 1995). La búsqueda de «calidad» en educación ambiental, la conciencia de que la actuación sobre el medio ambiente se mueve en otros ámbitos diversos de la mera acción técnica de dominio sobre el medio ambiente, impone en primer lugar una reflexión sobre el conocimiento. En este sentido se debe reconocer que la escuela inconscientemente ha asumido, en los contenidos y en los métodos, una visión del mundo que todavía considera posible el dominio de la naturaleza por el hombre y la previsión de los efectos futuros de acciones que actualmente ya tienen una implicación planetaria. Si analizamos no las últimas teorías científicas sino los «presupuestos de la vida cotidiana», entonces reconocemos que libros de texto, enseñantes y científicos comparten en gran medida esa cultura que un científico italiano, Marcello Cini, llama la cultura del maquinismo (1994). Esta cultura debe ser sustituida por una cultura de la complejidad, en la que se reconozca la interrelación y la contingencia de todos los fenómenos y, en consecuencia, su intrínseca imprevisibilidad.

Reflexionar sobre esta nueva cultura es una necesidad para todos los enseñantes, pero sobre todo para los docentes e investigadores que trabajan en el ámbito de la educación ambiental. De hecho, lo que se pone en tela de juicio no son las teorías ni las leyes de la cultura científica, sino las «creencias» que las acompañan y cuyos cambios son mucho más lentos y difíciles. El caos determinístico - por el cual pequeñas diferencias en las condiciones iniciales llevan a comportamientos tan dife- 
rentes que cada uno debe considerarse como un caso en sí mismo-, el efecto mariposa -por el cual variaciones mínimas, el batir de alas de una mariposa pueden extenderse y amplificarse en el espacio y el tiempo hasta provocar un tornado-, el principio de indeterminación establecido por la mecánica cuántica, el orden mediante fluctuaciones propuesto por Prigogine, el carácter absolutamente aleatorio de la evolución natural subrayado por Gould son todos ellos aspectos de esta ciencia de la complejidad que no es alternativa a la ciencia moderna, sino que, por el contrario, está bien implantada en el mundo científico y tecnológico.

El problema es que todavía pocas de las ideas y de los postulados de esta ciencia moderna han entrado a formar parte de la imagen popular de lo que es la ciencia. Científico es aún sinónimo de verdadero, y la característica principal del conocimiento científico es la de ser «cierto» y de proporcionar, por tanto, aquella «capacidad de previsión» que fascinaba a Laplace en 1700 , cuando junto con la Ilustración nacía el mito del progreso de la técnica. Decía Bateson (1991):

«Según la imagen popular de la ciencia, todo es, por principio, previsible y controlable; y si cualquier acontecimiento y proceso no es previsible y controlable en el momento actual de nuestros conocimientos, un leve aumento del conocimiento y, sobre todo, de la tecnología nos hará capaces de prever y controlar las variables rebeldes.»

Esta imagen de la ciencia está tan generalizada que contagia incluso las reflexiones sobre el medio ambiente y los problemas ambientales: más que tratar de entender, se intenta «resolver», a menudo afrontando los problemas por separado, como demuestra el ejemplo de la gasolina «verde» o el de los tubos de escape «ecológicos».

Reconocer que hace falta pasar de una cultura reduccionista a una cultura de la complejidad lleva a hacerse preguntas sobre los procesos de adquisición de conocimiento.

En la cultura ecologista se habla a menudo de enfoque sistémico y de «holismo», pero el reduccionismo no se encuentra tanto en el punto de vista disciplinar: Bateson afirma que cada conocimiento está construido y se ubica con respecto a la realidad como un mapa con respecto al territorio. El problema aparece en el momento en que se confunde el conocimiento disciplinar con la realidad, el mapa con el territorio.

Si cada experimentación científica, cada reconstrucción histórica, corresponde a una apuesta sobre la realidad (Stengers, 1988), la operación reduccionista prolonga la pertinencia y la validez de la apuesta más allá del campo en que ha demostrado ser fecunda. Se pretende descubrir las reglas que permitan decidir de una vez por todas qué resulta pertinente y significativo y qué accesorio y descuidable. Esta extensión indebida de estrategias, de modos de pensar, de modelos que se han demostrado eficaces en contextos concretos y para determinados fenómenos, hacia contextos y fenómenos similares sólo en apariencia, constituye la raíz misma del reduccionismo.

Y la vida cotidiana, la práctica escolar, las teorías pedagógicas están repletas de este fenómeno. Reducir el mundo a una máquina, reducir el cerebro a un ordenador, la escuela a un programa y la acción de los enseñantes a una programación significa no aceptar que estamos tratando con sistemas complejos, con individuos vivos, para los que cada acción es única y cada efecto nunca es completamente reproducible.

\section{Afirman Maturana y Varela (1990):}

«Sería $[. .$.$] un error definir el sistema nervioso como$ algo que tiene entradas y salidas en el sentido tradicional [...] el sistema nervioso no «toma información» del ambiente [...] sino que, por el contrario, gobierna la situación determinando qué configuraciones del ambiente son perturbaciones y qué cambios provocarán en el organismo. La metáfora, tan usada, de cerebro como ordenador no es sólo ambigua sino completamente equivocada.»

Y a pesar de todo, de manera más o menos consciente, es la metáfora más usada en el ámbito educativo: cuando se dice que se «comienza de nuevo la asignatura desde el principio» porque los estudiantes no han aprendido nada, se presupone que la experiencia de un alumno pueda ser cancelada como la memoria de un ordenador.

Incluso cuando se decide tener en cuenta las investigaciones actuales sobre «representaciones mentales de los estudiantes», o partir, para un aprendizaje significativo en el sentido de Ausubel (1978), de los conocimientos que el estudiante ya posee, a menudo no se presta atención a las operaciones reduccionistas llevadas a cabo por el enseñante y por el libro de texto, y de esta manera se dan por descontadas explicaciones y representaciones que en realidad son deducibles y comprensibles sólo en el contexto escolar.

Algunos ejemplos nos llegan justamente de las investigaciones sobre representaciones mentales (Mayer, 1990): Una chica, a la pregunta ¿Por qué la Luna no se cae sobre la Tierra?, responde: La Luna no puede caerse sobre la Tierra, ya que la Luna gira alrededor de la Tierra y no encima; aun, si cayese, no golpearía la Tierra, sino que pasaría al lado.

La chica no es tonta, y la respuesta es sólo un ejemplo de los procesos de construcción de los conocimientos que están teniendo lugar racionalmente sobre la base de otros conocimientos previos. De hecho, el sistema solar siempre se representa en los libros sobre un «plano horizontal». La vertical está caracterizada por la plomada, por tanto, en el sentido de caída de los pesos, en consecuencia, la Luna también debería caer sobre la vertical, al lado de la Tierra y no encima. El razonamiento relaciona, simplificándolos, conocimientos diversos, pero lo mismo hacen el libro de texto y los enseñantes. 
El cambio conceptual que intenta provocar la enseñanza científica no podrá nunca cancelar los obstáculos que radican en la experiencia, y que permanecerán como parte del propio bagaje personal de conocimiento (Bachelard, 1983). Pretender que algunas experiencias escolares puedan por sí mismas modificar un modo de ver el mundo es una vez más una pretensión reduccionista: lo que cabe esperar es conseguir «adjuntar» una nueva visión del mundo a la precedente, dar ejemplos conscientes de aquel cambio 2 (Watzlawick et al., 1974), en el que se cambian no sólo las estrategias con las que se juega, sino también las propias reglas del juego.

Otra nueva tentación reduccionista surge de la actual investigación pedagógica de los mapas conceptuales (Gowin y Novak, 1984) cuyo uso se ha generalizado no tanto con el fin de construir una conciencia de las relaciones entre los conceptos que cada uno individualmente crea, sino para replantear -y, en el mejor de los casos, valorar- las relaciones «justas», las jerarquías «correctas», el punto de vista, en definitiva, del libro de texto o del enseñante.

Ninguna de estas estrategias es en sí misma reduccionista, pero el uso que se hace de ellas a menudo sí lo es. Perder rigidez, transformar los mapas de Novak de diagramas jerárquicos en «redes» (Galagowsky, 1993) o en «laberintos» permite acercarse más a la complejidad de nuestros procesos de conocimiento. De esta manera Franco Lorenzoni (uno de los maestros que en Italia han reflexionado más sobre la complejidad del proceso de enseñanza) describe el proceso con el que sus niños han redescubierto la técnica de los mapas en la escuela elemental de Giove:

«A lo largo de todo el año coleccionamos intentos de encontrar modos de describir el espacio de las relaciones. A partir de un determinado momento comenzamos a rellenar folios y carteles con recorridos de palabras. Recuerdo el día en que, tumbados por el suelo, construíamos un cartel con mil ramificaciones que parecían no acabarse nunca. Fue entonces cuando Deborah propuso llamar a nuestros inciertos tentativos laberintos de relaciones.»

\section{Y en este sentido comenta:}

«He experimentado que los laberintos constituyen un estímulo eficaz para razonar: generan preguntas, suscitan problemas y se oponen a las múltiples simplificaciones que oprimen la escuela elemental.»

«Puede parecer paradójico y, sin embargo, estoy profundamente convencido de que la escuela y los adultos no deben simplificar la realidad cuando se la presentan a los niños. No deben esquematizar la variedad del mundo, porque de otro modo corremos el riesgo de reducir el conocimiento a bocados cocinados y ya masticados, sin sabor» (Lorenzoni, 1994, pp. 39-41).

Nuestra enseñanza, sea científica o humanística, es simplificatoria y reductiva, sobre todo porque no toma en consideración las evoluciones individuales y la raciona- lidad de los procesos a partir de contextos diferentes, fijándose únicamente en la certeza de los resultados.

La escuela tiende a trasmitir no los problemas sino las soluciones -las leyes «naturales», las fórmulas para calcular, la «correcta» interpretación histórica, los significados «apropiados» de los textos literarios-. La escuela «trasmisiva» necesita certezas que justifiquen su papel; el saber debe ser cierto, universal, objetivo. Pero incluso la escuela «constructiva», que actualmente representa la alternativa más avanzada, por un lado, declara que los estudiantes deben construir el propio saber, pero no se ofrece a sí misma como ejemplo de construcción crítica del saber, de un enfoque plural de las disciplinas. En este sentido, su objetivo parece ser el de reconstruir las mismas certezas (de la escuela de antes), y difícilmente acepta que se construyan saberes, y epistemologías, diversos a los codificados.

Un ejemplo de alternativas de concebir el saber se refiere justamente al modelo epistemológico de las ciencias naturales, que se puede identificar con aquél que un historiador italiano, Carlo Ginzburg, ha llamado el paradigma galileano (1984): descartar todo lo que es accesorio y contingente para poder generalizar y cuantificar.

Galileo investiga de esta manera, por medio de un proceso de simplificación de los fenómenos, aquellos elementos que se pueden reconocer idénticos bajo aspectos en apariencia diversos, que pueden ser reproducidos en el laboratorio y, por tanto, sometidos a verificación experimental, y que se pueden expresar matemáticamente. Ésta es todavía la imagen del modo de proceder de la ciencia que trasmiten los libros y los maestros. De este modo no sólo desaparecen las diferencias -aquéllas que constituyen el carné de identidad de cada fenómeno, de cada organismo, de cada proceso tomado de forma aislada-, sino que desaparece también el tiempo irreversible: los fenómenos que se presentan una sola vez, que no son idénticamente repetibles, no pueden ser comprendidos «científicamente».

A este modo de proceder, Carlo Ginzburg contrapone un modelo epistemológico que, aun de manera no explícita, se ha asentado en las ciencias humanas y, en particular, en la historia. El modelo se basa en presupuestos opuestos al galileano: lo que cuenta para comprender un hecho, para interpretar un fenómeno, son las diferencias, los pequeños indicios, las huellas, que permiten reconstruir un dibujo que, de otra forma, permanecería velado. Recuerda Ginzburg que un estudioso de arte italiano, Giovanni Morelli, reconocía la autoría de cuadros por los particulares más insignificantes de la pintura: uñas, lóbulos de la oreja, cabellos, que revelan -o sería mejor decir traicionan- la mano del autor y permiten, en consecuencia, distinguir un original de una copia.

Carlo Ginzburg propone el reconocimiento en este modo de proceder de un método de tipo diverso, que él llama paradigma indiciario. Ese método no tiene nada de irracional ni de ilógico; por el contrario, constituye un acercamiento racional hacia un mundo hecho de diferencias y cambios, en el cual «construir historias», que sean 
al tiempo coherentes internamente y adecuadas a los indicios que se tienen a disposición, permite sacar a la luz significados que, de otro modo, permanecerían ocultos.

El paradigma indiciario tiene raíces profundas en la cultura popular: los cazadores que reconocen por las «huellas» los animales que persiguen y su comportamiento; los viejos campesinos que saben predecir el tiempo basándose en sutiles diferencias del paisaje y del comportamiento de los animales; los médicos -no los «especialistas» actuales- que reconocen las señales, los síntomas, de un malestar general. La literatura está llena de ejemplos de paradigma indiciario -del Zadig, de Voltaire, al episodio que introduce Fray Guillermo en El nombre de la rosa, de Umberto Eco.

En todos estos casos, las historias que se reconstruyen son totalmente únicas, casuales, no repetibles, no previsibles; y de ahí que su «previsión retrospectiva», su reconstrucción, provoque asombro. La geología, la paleontología, la física del big-bang están en realidad mucho más cercanas a este tipo de racionalidad que a la propuesta por el paradigma galileano. Por su parte la biología participa de los dos paradigmas: ciencia experimental en lo que se refiere a los aspectos generales reconocibles en todos los seres vivos, y ciencia histórica en lo relativo a la evolución biológica y de las especies $\mathrm{y}$ de los individuos.

Las ciencias ambientales, pues, suponen un continuo de problemas «imprevisibles», pero, a posteriori, perfectamente explicables. "Quién habría pensado en los años treinta que la introducción del tractor en la agricultura haría aumentar los casos de cáncer y el precio del pescado?», se preguntaba una notable ecologista italiana, Laura Conti (1989). Y, ¿hasta qué punto eran previsibles los desastres causados a la economía egipcia por la presa de Asuán o el agujero en la capa de ozono? ¿Cómo se puede enseñar a prever lo imprevisible?

El paradigma indiciario puede ser útil en la educación ambiental, ya que pensar en historias, enlazar, a través de relaciones, elementos anteriormente identificados como diferentes es la manera natural con la cual exploramos «la pauta que conecta» (Bateson, 1982) las diversas partes de nuestra realidad. Es el modo a través del cual establecemos contextos y construimos significados; es nuestra manera de explorar las relaciones posibles.

Las historias permiten construir contextos y significados incluso cuando la lógica, y en particular la lógica aristotélica, parece impedirlo. Las historias permiten, por tanto, un concepto de racionalidad más flexible, en el cual las reglas del juego se pueden trastrocar intencionadamente para así poder explorar caminos diversos. También las conquistas científicas de nuestra época pueden ser consideradas «historias» (Stengers, 1992): historias, como las de la revolución copernicana y newtoniana que han acumulado en el tiempo indicios e interpretaciones tales como para ser consideradas irreversibles, pero incluso historias que podrían todavía ser modificadas, como el enfoque mecanicista de la medicina hacia el cuerpo humano o de la ingeniería genética hacia el ser vivo. Historias peligrosas que no sólo exploran otras posibilidades, sino que crean el mundo que están imaginando.

\section{EDUCAR SIN CERTEZAS}

Dentro de la investigación ENSI, enseñantes e investigadores de diversos países europeos han discutido entre ellos y con expertos internacionales sobre la relación existente entre educación en la complejidad y educación ambiental (OECD, 1995). De esta manera intervenía Franco Lorenzoni en un congreso organizado en Perugia en 1992:

«Hay un isomorfismo entre medio ambiente y relación educativa, lo cual es ventajoso. Nosotros podemos decir que la escuela, respecto a algunas disciplinas, quizá es más eficaz adoptando un sistema lineal. Quizá, ¿por qué no? Pero, si en la escuela queremos proponer una educación en el medio ambiente, estamos absolutamente obligados a actuar de una manera compleja. Desde este punto de vista surge un desafío que el objeto mismo del conocimiento lanza a los educadores. No es un problema de coherencia, es una cuestión epistemológica. Pero nos encontramos con el problema de la fatiga: trabajar en la relación educativa en términos de complejidad conlleva una extraordinaria fatiga, una continuo afrontar obstáculos. Quiero poner un ejemplo: la escuela actúa en cierta medida como la agricultura química; para obtener el trigo aporta todos los aditivos que son necesarios para que haya trigo y para eliminar todo lo que no es trigo. La escuela de este modo funciona bastante bien, como por su parte la agricultura química: da de comer a las personas. El problema reside en el desastre que crea a largo plazo, porque no se da a la tierra los recursos necesarios para recrear la productividad. Y esto mismo hacemos con los niños con los que trabajamos: a menudo reducimos drásticamente sus posibilidades de elegir cambiar su punto de vista y afrontar de manera activa la incertidumbre.»

Reducir la incertidumbre, simplificar el mundo para que sea mecánico y previsible [comporta una reducción incluso de la creatividad]; reducir la redundancia para mejorar la eficiencia reduce también las posibilidades y, por tanto, las capacidades de adaptación. La cultura del maquinismo ha creado la ilusión colectiva de la posibilidad de conocimiento ilimitado y de control absoluto: parece que sea sólo cuestión de tiempo, de personas o de fondos suficientes. Sin embargo, a cada aumento de conocimiento, corresponde un aumento de la toma de conciencia de nuestra ignorancia, y este proceso no puede tener fin.

Los conocimientos construidos hasta el momento no pueden verse como leyes determinísticas, o como modelos, de una realidad que se mantiene invariable en el espacio y el tiempo. El paraíso de las certezas científicas se ha perdido para siempre: 
«Creíamos vivir en un universo regido por leyes necesarias y eternas en el que bastarían conocimiento y razón para proyectar y hacer posible un futuro cada vez mejor. Nos encontramos, sin embargo, en un mundo con procesos evolutivos irreversibles en el cual se entrecruzan casualidad y necesidad, donde hace falta sobre todo sabiduría, prudencia y solidaridad para poder afrontar las consecuencias imprevistas de nuestras elecciones y para no hacer recaer irresponsablemente sobre las generaciones futuras el peso de nuestras deudas. El paraíso en el que creíamos vivir ha desaparecido: cuanto antes nos demos cuenta, mejor» (CINI, 1994).

El problema principal en un mundo que ya no es mecánico y previsible es el de aceptar convivir con la incertidumbre. La necesidad de certezas, aunque sean limitadas en el espacio y el tiempo, es, por su parte, una exigencia imprescindible de la naturaleza humana. El problema no es, por tanto, renunciar a todas las certezas, sino reconocer los niveles en los que dichas certezas se pueden buscar.

El papel de los «expertos», ya sean enseñantes o investigadores, debe, pues, cambiar; los expertos deben aprender a no ser los que conocen las respuestas sino sobre todo los que son capaces de formular preguntas y de discutir las maneras a través de las que hay que buscar las respuestas. De esta forma, hace unos años, algunos «expertos» describían el papel que la ignorancia puede tener en nuestra sociedad

«La perspectiva filosófica [...] es la de la complementariedad entre el saber como objeto (knowing-that) y el saber como proceso (knowing how), en la que la incertidumbre y la cualidad son atributos esenciales del conocimiento, y en la que la interacción entre conocimiento e ignorancia es dialéctica. De este modo, la ignorancia se hace utilizable y, más aún, útil y esencial, para la comprensión de nosotros mismos y de nuestras relaciones con el ambiente. La conciencia de la ignorancia puede constituir el principio de una nueva sabiduría con respecto al lugar que nos toca en el mundo contemporáneo» (Ravetz y Funtovicz, 1989).

Esta conciencia, esta sabiduría, es justamente lo que se echa de menos en muchos programas de educación ambiental todavía hoy. Hablar de actuación en pro del medio ambiente, de desarrollo -incluso sostenible-puede precisamente invitar una vez más a simplificar, a reducir, para encontrar rápidamente aquellas soluciones «seguras» que garanticen la eficacia de la acción. La revalorización de la incertidumbre, de la duda y del conflicto debe ser, sin embargo, un elemento asumido por las escuelas que se implican en la educación ambiental en forma de conciencia que permita profundizar en los problemas, y afrontarlos, sin reducirlos o simplificarlos (Mayer, 1995).

El reto para los enseñantes consiste en transportar esta «revalorización de la incertidumbre» a la práctica educativa cotidiana. Stenhouse, en 1975, decía que a los enseñantes innovadores hay que pedirles que sean capaces de «soportar el fardo de la propia incompetencia» (taking on the burden of incompetence). Y este hecho, el poder soportar no ser experto y competente, es al mismo tiempo una de las mayores dificultades para el enseñante y una absoluta necesidad para la educación ambiental. Para el enseñante que quiere hacer educación ambiental no existen «seguridades», reglas que seguir. O mejor aún, la seguridad se traslada desde la seguridad de los resultados a la seguridad sobre la validez y la significación de los procesos puestos en marcha.

Es precisamente el reconocimiento de la propia «incompetencia» como expertos (y de la propia competencia como educadores) lo que permite a los enseñantes ser más flexibles, escuchar más las voces y las propuestas de los chicos, implicarse en un proceso de «co-creación» de proyectos, y de «co-evolución» de los procesos didácticos, que, aun siendo fatigoso, parece ser el único camino viable. En este sentido, los mismos enseñantes del proyecto ENSI comentaban este cambio de rol.

«Mi costumbre de querer tener todo bajo control, de programar y «hacer trotar» a los estudiantes y también mi formación científica determinística se dirigían exactamente contra la necesidad de practicar la capacidad de escuchar, de guiar la reflexión, de encontrar el modo de "observarme a enseñar", de aceptar el punto de vista de los estudiantes como un elemento fundamental de nuestro diálogo» (Luisa Borettini, en Losito y Mayer, 1995).

«El enseñante no es un libro que conserva conocimientos inmutables, sino más bien un marinero que se adapta cada vez a las condiciones del mar» (Carlotta Lazzarini, en Losito y Mayer, 1995).

«Libertad en la incertidumbre. Yo no sabía dónde nos llevaría. En algunos momentos me he sentido perdida. Después, poco a poco, el proceso se ha puesto en marcha, los alumnos tenían tantas ideas y energía. Mi papel era diferente pero empezaba a gustarme[...]» (Pozarnic et al., 1993).

Aceptar la incertidumbre como componente del conocimiento quiere decir poner en tela de juicio varios elementos de «sentido común» de la práctica docente, como, por ejemplo, el significado de una transmisión tout court del conocimiento, la posibilidad de definir objetivos educativos válidos para todos los alumnos en cualquier lugar o la tarea de la escuela de estandarizar y uniformar los resultados en función de las necesidades decididas por la sociedad.

En este sentido, von Foerster, uno de los padres de la cibernética, ridiculiza los sistemas de evaluación «objetiva»:

«Consideremos el modo de funcionar de nuestro sistema educativo: en el momento en el que el estudiante ingresa en el sistema escolar, éste es una imprevisible "máquina no banal". No sabemos qué respuesta dará a una determinada pregunta. Pero, si al final obtiene los resultados que el sistema se espera de él, las respuestas que dará a nuestras preguntas deberán conocerse de antemano. Los tests escolares son un medio para medir el grado de 
banalización: el estudiante es completamente previsible $\mathrm{y}$, por lo tanto, puede ser admitido en la sociedad. No será fuente de sorpresas, ni de problemas» (von Foerster, 1987).

La educación ambiental nos permite poner en evidencia la contradicción interna presente en cualquier programa educativo: por una parte, es necesario construir un «lenguaje común y homogéneo» y un mínimo de competencia; por otro lado, «respetar las diferencias» no puede ser un eslogan a aplicar sólo a la biodiversidad, sino que debe poderse aplicar también a los estudiantes.

Es interesante también ver cómo el papel de la escuela en el mantenimiento de este equilibrio entre necesidad de homogeneización y necesidad de mantener la diversidad se está modificando con el tiempo. La sociedad, de hecho, ha encontrado en estos últimos veinte años nuevas maneras y nuevos sujetos que garanticen la normalización y reduzcan las diversidades, mucho más eficaces que la escuela: la televisión y, en general, los medios de comunicación de masas y la internacionalización del mercado. Las diferencias o se agravan -entre religiones, etnias, culturas profundamente diversas- o se reducen todos los estudiantes del mundo ven las mismas telenovelas, llevan las mismas zapatillas, se reconocen en los mismos modelos de vida-. El papel de la escuela para el futuro parece ser, por tanto, el de garantizar el desarro1lo, y la aceptación de las diferencias individuales más que el de su abolición; el de permitir a cada uno encontrar su propio camino autónomo e independiente del conocimiento, si bien fundado en una base común.

Una posible vía es la de considerar la escuela como el lugar donde se hacen preguntas legítimas (von Foerster), es decir aquellas preguntas de las que no se conoce la respuesta, y para las que cada uno es libre de elegir los modos de actuar y las informaciones que le parecen oportunas. Cuando se habla de enfoque constructivista en la enseñanza de las ciencias, «como tratamiento de situaciones problemáticas abiertas que los alumnos puedan considerar de interés» lo que se plantea es un aprendizaje (Gil et al., 1991), pero muchas veces lo que se lleva a la práctica son propuestas de las preguntas de siempre, alejadas de los contextos reales de la vida de los chicos, y cuya respuesta es única, ya conocida por el profesor. En la educación ambiental no es difícil encontrar «preguntas legítimas» que hacerse y que hacer a los alumnos, y el conocimiento puede ser ciertamente construido conjuntamente, entre profesor y alumno. Lo que se obtiene no es una simulación del trabajo de los científicos, es realmente un conocimiento nuevo del que previamente no se disponía: conocimiento local y contextualizado, del cual no se extraen leyes generales sino indicaciones para la acción.

\section{CONSTRUIR VALORES}

Afirmar la complejidad de la realidad no quiere decir, pues, renunciar a conocerla, sino asumir la responsabilidad de un conocimiento que pretende aunar, en una «única historia», las informaciones y los valores, las sensaciones y las interpretaciones; un conocimiento que ya no es inmutable y objetivo, que es consciente de que

«No existe una lectura de lo existente, sobre todo si se trata de situaciones que afectan a los seres humanos y a su ambiente, que no sea ya implícitamente interpretativa, y que prepare o comporte, de manera implícita, elecciones o juicios de valor» (Stengers, 1992).

Y que requiere, por tanto, asumir la responsabilidad

«[...] por la manera en que somos capaces de definir el mundo, de tenerlo en cuenta en nuestras experiencias» (Stengers, 1992).

El problema es, pues, el de introducir en la escuela la confrontación crítica, incluso sobre temas controvertidos, sobre el análisis y la explicitación de los valores subyacentes. La discusión sobre los diferentes puntos de vista y los diversos modos de resolver un problema es una práctica que empieza a generalizarse en una escuela que se define constructivista. Pero la educación ambiental necesita ir más allá: requiere poner en tela de juicio los valores implícitos que aún están presentes en nuestra escuela. Cada disciplina, cada libro de texto, transmite, de hecho, mensajes relativos a una visión del mundo: la ciencia se ocupa de lo que es «verdad», la técnica de lo que es «útil», el arte de lo que es «bello», la economía de hacer crecer «el bienestar». Y, a través de estas definiciones implícitas de «valor» y de la potencialidad de las disciplinas, se transmiten modelos de comportamiento y de juicio. Incluso actualmente el énfasis de todas las disciplinas se centra en el progreso y el desarrollo, frecuentemente entendido en manera cuantitativa; todavía hoy bien poca atención se ofrece a las culturas y a los puntos de vista de los otros pueblos. Nuestra escuela es aún una escuela que prepara para vivir en un «mítico farwest», con espacios y recursos ilimitados donde el individualismo puede ser la clave del éxito, y no inmersos en un «planeta-astronave», con recursos limitados, donde sólo la cooperación y el respeto hacia el prójimo pueden evitar el desastre.

Los valores, por tanto, deben en primer lugar «descubrirse», sacarse a la luz, para poder examinarlos y poner en evidencia las contradicciones, que a menudo se introducen en las personas. En una conferencia internacional sobre «Valores en la Educación Ambiental» que tuvo lugar en Escocia en 1993, Peter Posch distinguía entre valores «expuestos» (espoused) y valores realmente utilizados (in use), y hacía ver que esta distinción permitía explicar parte de las dificultades que se encuentran en la educación de los valores.

«Si los valores que se han transformado en comportamientos son en gran medida inconscientes e indiscutibles es comprensible que no se cambien por los valores expuestos (aquéllos de los que discutimos y hablamos)» (Posch, 1993, p. 29).

Las personas, los estudiantes, los enseñantes, son víctimas de la misma esquizofrenia que azota nuestra socie- 
dad: los valores expuestos son los que resultan aceptables en el ámbito formal, aunque no correspondan a los valores que guían los comportamientos. Una manera de afrontar el problema es aceptar que, incluso, los valores pueden ser construidos, como los conocimientos y junto a los conocimientos de que forman parte, y que la escuela pueda ofrecer ocasiones para construirlos. Esto significa que, como para cada operación de cambio profundo, no se pueden tomar atajos, no se puede pensar que una acción colectiva, un comportamiento impuesto (por ejemplo, el reciclaje o la recogida selectiva) se convierta automáticamente en un valor aceptado y presente en la vida cotidiana. Hay que ofrecer tiempo y ocasiones para discutir y reflexionar: hay que renunciar a cambiar los comportamientos en tiempos breves con el fin de intentar crear las condiciones para un cambio más a largo plazo.

En esta situación, el papel del enseñante es muy difícil: por un lado, no puede no explicitar sus propios valores; por el otro, debe respetar los de los chicos. La dificultad de acción en la que se encuentra el enseñante que se implica en la educación ambiental, como en cualquier otra «educación» que tenga en cuenta los valores, es la de

«[...] creer en lo que se hace y al mismo tiempo dar espacio a otras creencias» (Caiani y Ciancaglini, en Losito y Mayer, 1995).

El mismo problema afrontaba Stenhouse en los años setenta con su Humanities Project (1971, 1975): no pudiendo eliminar los valores, los prejuicios, la única solución es sacarlos a la luz, ser conscientes de las diferentes maneras de interpretar un hecho concreto. Para Stenhouse, esta conciencia se puede construir sólo si el enseñante respeta algunos principios metodológicos (Elliot, 1985):

- la actividad principal en el aula es la discusión y no la instrucción;

- hay que salvaguardar la diversidad de puntos de vista;

- el rol del enseñante debe inspirarse en una «neutralidad metodológica»;

- los enseñantes tienen la responsabilidad de la calidad del aprendizaje y, por tanto, de presentar los criterios con los que discutir y criticar los puntos de vista.

Precisamente la contradicción en la que se encuentra el enseñante que hace educación ambiental está en cómo mantener esta «neutralidad metodológica», un equilibrio entre sus valores «ambientales»y sus valores «educativos», sin promover o bien opiniones y actuaciones distintas de las que considera justas o bien «adiestramientos» en sus valores. De este modo, un enseñante «ecologista» expone la posición a la que ha llegado fatigosamente:

«Creo que una gran apuesta de la educación ambiental es conseguir no hacer ecologismo. Sería muy limitante o, más aún, podría tener efectos contraproducentes una acción educativa encaminada a crear hábitos de respeto hacia el ambiente.

»Uno de los "retos" más importantes de la educación ambiental es, en mi opinión, justamente el de educar en la confrontación de valores, analizados desde diversos puntos de vista, y en relación a un problema concreto. $\mathrm{Si}$ los estudiantes saben valorar la complejidad de los temas ambientales, si han adquirido un método de análisis de las "posiciones en el campo", podrán realmente ser libres y capaces de elegir una posición propia, comprender y «desvelar las razones no formuladas» (de orden político, económico, etc.) que están detrás de la toma de postura por parte de los diferentes sujetos que se confrontan en un problema» (Rodolfo Vezzosi, en Losito y Mayer, 1995).

La puesta en juego no es, efectivamente, una adhesión crítica a los valores llevados adelante por el ecologismo, ni es la aceptación de un nuevo dogmatismo, aunque sea de signo diferente del que actualmente domina, sino la capacidad de discutir críticamente soluciones y propuestas, de confrontarse con los llamados expertos, de exigir que se tengan presentes los puntos de vista y las dimensiones a las que somos sensibles. Como decía Isabelle Stengers al final de su intervención en Perugia (1992), lo que con la educación esperamos alcanzar es:

«[...] la invención de prácticas realmente democráticas, las únicas capaces de dar un sentido creativo, crítico y constructivo a la noción de complejidad.»

\section{INVESTIGACIÓN Y ACCIÓN}

Reflexiones del tipo de las que se han hecho hasta ahora han acompañado, a veces explícitamente, con más frecuencia implícitamente, una práctica de la educación ambiental que se caracteriza por ser profundamente innovadora respecto al conjunto del sistema educativo, si bien el riesgo de caer en el tecnicismo y en el reduccionismo está siempre presente.

En muchos países, el desarrollo del concepto y de los objetivos de la educación ambiental ha venido de la mano de una evolución de la organización escolar, desde propuestas de trabajo y de reformas, incluso estructurales, que han tratado de resolver las dificultades y las contradicciones que se han ido encontrando:

- La propuesta de «temas transversales» ha intentado en muchas naciones europeas dar respuesta a la contradicción entre la necesidad de dar un espacio propio a la educación ambiental y la naturaleza intrínsecamente interdisciplinar y «transversal» de los conocimientos que ésta propone.

- Las semanas verdes, las excursiones naturalistas, el trabajo en la naturaleza han llegado a formar parte, en casi toda Europa, de las actividades escolares normales, al menos, en la escuela obligatoria. 
- El proyecto educativo de centro se está afianzando como uno de los instrumentos capaces de conciliar la sustancial homogeneidad de los currículos con la necesidad de diversidad local: la educación ambiental encuentra, en este ámbito, espacio para proyectos de clase, transversales y multidisciplinares, casi siempre referidos a actuaciones, investigaciones e intervenciones en el territorio circundante.

- A medida que la escuela se abre a los problemas del medio ambiente, se abre también a la colaboración con las instituciones externas implicadas, dando lugar de este modo al trabajo conjunto entre escuela y empresa y entre escuela e institución, a través de «partenariados» que son instrumentos de estudio y de actuación al mismo tiempo.

Estos cambios no son cambios superficiales, porque lo que está en discusión no son los contenidos del aprendizaje, lo que se debe conocer, sino cómo se debe conocer y por qué.

La educación ambiental, junto a las otras educaciones transversales que afrontan los temas de la ciudadanía en el mundo actual, está modificando la concepción misma de escuela y de educación.

La educación ambiental es, efectivamente, «transversal» no sólo porque puede ser asumida por todas las materias escolares, sino porque intenta relacionar diversos tipos de reflexiones: aquella más ecológica coherente con la epistemología del conocimiento sobre la naturaleza; aquella más metodológica coherente con la complejidad de este tipo de conocimiento (Morin, 1994); y aquella más específicamente pedagógica coherente con los conocimientos actuales sobre los procesos educativos y de aprendizaje. Además es transversal porque, a diferencia de la mayor parte de las disciplinas escolares, logra poner sobre una única mesa puntos de vista e intereses diferentes: desde asociaciones ecologistas a enseñantes que quieren construir la innovación, a entes locales que buscan responder a las exigencias y a las emergencias ambientales, a ciudadanos comunes que se preocupan de estas emergencias.

Mediante la educación ambiental, la imagen de la escuela y del aprendizaje se transforma: $a$ ) de una escuela que transmite conocimientos elaborados en ámbitos externos en una escuela que construye conocimientos relevantes en el ámbito local; $b$ ) de una escuela cuyos objetivos están vinculados casi exclusivamente a los conocimientos a una escuela que quiere involucrar los sentimientos, discutir los valores, inventar nuevos comportamientos; $c$ ) de una esuela estática que se modifica tardíamente según los estímulos de la sociedad a una escuela que quiere modificar la sociedad, y que no acepta un rol subalterno respecto a otras instituciones (Elliott, 1991; Posch, 1991). Y junto a la imagen de la escuela, se transforma también la imagen de la investigación educativa, del conjunto de operaciones que guían y hacen posible una reflexión acerca del camino que se está haciendo. Mientras los docentes se encuentran asumiendo un rol de investigadores, los «investigadores» deben construirse un rol de «mediadores» entre la investigación más general y académica y la investigación en la práctica de los enseñantes que construyen día a día, mediante una reflexión sobre la acción, su propio camino.

En efecto, es indispensable que, junto a la experimentación y a la innovación, se desarrolle una investigación y una reflexión sobre el significado de lo que se está proponiendo, sobre la «epistemología»-para decirlo como Bateson- en que se basa la innovación. Sin esta reflexión, la educación ambiental corre el riesgo de caer en una práctica de rutinas, de transformar una «aventura en un territorio desconocido», en el que sabemos que no podremos nunca volver sobre nuestras propias huellas, en una «visita guiada», siempre previsible y sin interés. En este sentido, si de verdad quiere ser coherente con sus finalidades, la educación ambiental no puede renunciar a ser una disciplina de «frontera», lindante entre estructuras disciplinares y puntos de vista globales, entre la seguridad sobre los valores que la guían y sobre los procesos que quiere poner en práctica y la incerteza sobre los itinierarios y sobre los resultados concretos que cada iniciativa deberá construir a la largo del camino.

La reflexión sobre la acción es uno de los elementos que caracterizan el proceso hacia un cambio profundo que ponga en discusión las imágenes difusas de lo que es y debe ser la educación. La reflexión sobre la acción, la investigación en la acción, permite, en efecto, actuar y tomar decisiones aun en la incerteza, controlar el propio itinerario y modificarlo rápidamente si es necesario, adaptándose a los «imprevistos» y aceptando una «coevolución» entre contexto educativo y sujeto que aprende. La investigación-acción permite a los enseñantes, a los estudiantes y a las estructuras aprender de la experiencia y modificarse conjuntamente.

Por ello, la investigación-acción ha sido experimentada como metodología de investigación en diversos programas de educación ambiental a partir del proyecto ENSI dell'OECD, un proyecto en el que han participado más de 200 enseñantes de 19 naciones (OCDE, 1995). Se propuso a los enseñantes utilizar las iniciativas para la educación ambiental que estaban poniendo en práctica en sus clases como contexto en el cual formularse preguntas «legítimas» sobre cómo desarrollar en los alumnos la sensibilidad por el ambiente y las «capacidades dinámicas» (Posch, 1991), es decir, capacidades de autonomía, de responsabilidad individual y social, de solidaridad. El resultado fue, para muchos de los docentes que participaron, la puesta en discusión del propio rol, de la imagen de lo que debe ser la escuela, de las disciplinas y la búsqueda de procedimientos y de diferentes maneras de construir conocimientos (Mayer, 1995).

El conocimiento fue construido «localmente» por los estudiantes y los enseñantes conjuntamente, para responder a las exigencias del problema estudiado (Posch, 1991), pero los grupos de enseñantes que se reunían periódicamente en el ámbito nacional construyeron, a su vez, colectivamente, un «conocimiento sobre el conoci- 
miento», una epistemología explícita respecto a las tantas epistemologías implícitas enraizadas en nuestro sentido común.

El enseñante, por tanto, se transformó en investigador, como proponía Stenhouse en 1975, mientras que el investigador institucional, el «soporte pedagógico» previsto por la investigación, ha desarrollado, esencialmente, un rol de facilitador de la reflexión, de animador de los grupos de trabajo, de responsable de las metodologías utilizadas, de mediador entre los diversos valores y las diversas metodologías que surgían en el curso de la investigación (Mayer, 1997a).

La investigación-acción constituyó, a través de la investigación ENSI, un ejemplo de un contexto de formación de enseñantes no dirigida a través de un proceso «en cascada» desde lo «alto»-la universidad, el ministerio o el centro para enseñantes- hacia lo «bajo», sino horizontalmente, a través de un proceso «de contagio», en el cual todos contribuyen a la formación y todos construyen la propia formación, incluso los investigadores universitarios (Losito y Mayer, 1995). En un proceso de investigación-acción, en efecto, los dos momentos, el de la innovación y el de la formación, no pueden estar separados: «desarrollando el currículo a través de la investigaciónacción sobre contextos particulares de aprendizaje, los enseñantes se desarrollan a su vez, ellos mismos, en su rol profesional» (In developing the curriculum through action research in particular learning contexts, teachers also develop themselves within their professional role) (Elliot, 1995).

La investigación-acción no es, seguramente, la única metodología de investigación que puede aplicarse a la exploración de los problemas y posibilidades de la educación ambiental, pero constituye un ejemplo de coherencia, de congruencia, entre los métodos de investigación de ámbito educativo y de reflexión y la complejidad propia de la educación ambiental. Seguramente, la investigación-acción tal como se entiende en el proyecto ENSI no permite a los enseñantes, y tampoco a los investigadores, encerrarse en las «cuatro paredes de la propia clase» (Menter, 1996, discutiendo las posturas de Elliot, 1995), sino que, al contrario, abre la confrontación entre puntos de vista y experiencias muy diferentes de las propias (por dar sólo un ejemplo, en la investigación italiana han trabajado conjuntamente, discutiendo sus proyectos de educación ambiental, enseñantes de todos los niveles escolares, desde la escuela infantil a las superiores; los intercambios más interesantes han sido, justamente, aquéllos entre docentes que, en general, no tienen ocasión de trabajar juntos, es decir, entre los enseñantes de educación infantil y primaria y los de la escuela superior).

La búsqueda de coherencia entre los principios y valores que guían la educación ambiental y los principios que guían la investigación educativa se va extendiendo a otras problemáticas pedagógicas y, en particular, a la relativa a la evaluación de proyectos de educación ambiental. Jan Robottom, ya en 1988 denunciaba, como relacionadas a un «paradigma botánico y agricultor» todas aquellas formas de evaluación que intentaban «separar las variables» y «evaluar los resultados» de un proyecto de educación ambiental, mediante el simple suministro de test. Evaluar un proyecto educativo es algo mucho más complejo que «medir» si un conocimiento ha sido adquirido o no, y la educación ambiental, que pretende ser flexible, transversal, explicitar valores y aceptar lo incierto e imprevisible, requiere procesos de evaluación articulados y respetuosos de los diversos puntos de vista. Un propuesta italiana, que estimulada por el proyecto ENSI, ha sido de interés para diversas realidades italianas y extranjeras, es la de definir, a través de un trabajo de investigación-acción que involucra a enseñantes e investigadores, los «indicadores de calidad», los «criterios» que, de algún modo representen las características de «un buen proyecto de educación ambiental» (Ammassari y Palleschi, 1991; Mayer, 1994). Estas características pueden ser usadas por los enseñantes, no sólo para evaluar el propio proyecto, sino también como «guía para la elaboración», como puntos de referencia. La propuesta italiana consideraba diez indicadores, subdivididos a su vez en índices, pero, naturalmente, las características deben adaptarse, necesariamente, al país en el cual se propone el proyecto y a la fase específica que esté pasando la educación ambiental en ese país, y sería interesante explorar cuáles y cuántos son los elementos comunes en el ámbito europeo o mundial.

El debate sobre la evaluación de los proyectos y de los programas propuestos a las escuelas por las autoridades escolares o por los entes públicos o privados, desarrollados, sobre todo, en los países anglosajones a partir de la mitad de la década de los años setenta, no se refiere sólo a los límites del enfoque experimental clásico sino que explora la posibilidad de enfoques diversos: desde un enfoque democrático (MacDonald, 1974), en el que el evaluador responde a la comunidad en su totalidad, reconoce la existencia de una pluralidad de valores y trata de presentar los puntos problemáticos del proyecto, hasta una evaluación de tipo responsable (Stake, 1988), en la cual el objetivo es proporcionar informaciones útiles, sobre todo, a los centros y a las personas que participan en el programa, pasando por un enfoque de tipo constructivista más que responsable (Guba e Lincoln, 1989), en el cual el énfasis se pone en el rol activo que deben asumir todos los interesados en la investigación en la construcción de la evaluación y en el rol esencialmente negociador entre los diversos intereses que deben ser asumido por el evaluador.

En Italia el observatorio nacional ORMEA ha tratado de aplicar este tipo de enfoques a los proyectos y a las inciativas para la educación ambiental puestas en práctica por las autoridades locales (Losito, 1996), y también a los proyectos internacionales financiados por la Unión Europea (Mayer, 1997b) o por asociaciones como el WWF (Mayer, 1997c). Actualmente, una red de investigación europea, la red REVERE (Reseau sur 1'Evaluation dans l'Education Relative à l'Environnement) está explorando las formas de evaluación epistemológicamente cohenrentes con la educación ambiental. 
La educación ambiental, por tanto, también mediante la investigación, está prefigurando nuevos modos de organizar, ya sea el aprendizaje, la formación, la evaluación. Se habla mucho, hoy en día, de redes, entendiéndose por ello «redes telemáticas», y se olvida que algunas de las primeras redes de intercambio y de colaboración desarrolladas en el ámbito internacional han sido y son de educación ambiental: desde el proyecto Green, proyecto internacional sobre el análisis del agua, al mismo proyecto ENSI; desde el proyecto Science across the Word, esponsorizado por la BP, al proyecto Globe lanzado por el vicepresidente de los Estados Unidos, Al Gore.

Una característica de estas redes es la dinámica de las relaciones, la colaboración y el intercambio, que se transforman en instrumentos para hacer frente a la complejidad y a la incerteza de las situaciones concretas. Estas «redes dinámicas» (Posch, 1995), por una parte, superan los límites entre escuela y sociedad, entre estudiantes y enseñantes y entre enseñantes e investigadores y expertos $y$, por otra parte, permiten a enseñantes innovadores poder contar con una «red de protección y soporte» que les ayuda a superar las dificultades que interpone siempre la escuela a la innovación y, al mismo tiempo, construir una comunicación interpersonal y crear relaciones de confianza duraderas. Son redes en las cuales no hay jerarquías fijas, sino que todas las posiciones son simétricas: las decisiones son contratadas o negociadas; el intercambio es contínuo y provechoso para todos y, sobre todo, la red es percibida por todos como un elemento de soporte y seguridad para afrontar la incerteza cotidiana. La atmósfera que se crea, la confianza, el espíritu de grupo y, al mismo tiempo, la apertura al exterior son los elementos característicos de este tipo de redes.

Estos instrumentos, investigación-acción, evaluación «responsiva»y redes dinámicas, son intrumentos que garantizan una reflexión sobre la educación ambiental aun cuando se utilizen fuera del contexto escolar. En Italia, desde 1994, el Ministerio del Ambiente está intentando construir un sistema nacional para la educación ambiental (Briano, 1996; Patriarca, 1995), sistema que se apoya en una red de centros de educación ambiental territorial, nuevas estructuras que deben operar sobre el territorio, en colaboración con los entes locales y con la contribución de asociaciones ambientalistas, para desarrollar un trabajo de mediación entre las propuestas de educación ambiental y las necesidades de las escuelas y, más en general, de los ciudadanos. Hasta ahora se han formado más de 30 laboratorios en treinta provincias italianas, y se espera que en breve existan noventa -uno por cada provincia- vinculados por una red -la red Labnet a cargo, experimentalmente, del Istituto di Tecnologie Didattiche del CNR, Consiglio Nazionale delle Ricerche, que es a la vez una red telemática de comunicación y de información recíproca, y una red de soporte y de formación (Briano, Midoro, Trentin, 1996a y 1996b). También forman parte de este sistema el Observatorio Nacional- ORMEA, a cargo del Centro Europeo dell'Educazione, que asegura una comunicación y una reflexión respecto a las metodologías usadas y a las investigaciones desarrolladas sobre la educación am- biental, y un archivo nacional -ANDREA, a cargo del Reparto di Psicopedagogia del CNR, dirigido por Francesco Tonucci- que está recogiendo no sólo la información relativa a los entes que se ocupan de educación ambiental y a los materiales producidos (Fichera, Marcheggiano, Mazza, 1996), sino también, bajo forma de hiperfichas, las experiencias y las reflexiones más significativas. (La dirección para buscar en Internet información sobre el sistema nacional italiano es: http:// www.labnet.cnuce.cnr.it, y para consultar el archivo http://www.psicoped.rm.cnr.it).

En el territorio nacional italiano la investigación-acción ha sido propuesta a varios ámbitos: en los centros de educación ambiental o redes que se han constituido en el nivel regional como metodología de investigación para los enseñantes, en el ámbito de las personas de los centros de educación ambiental como instrumento para reflexionar en grupo sobre las características de la propia profesión: una profesión nueva, en cuanto que no se trata de equipamientos que proponen actividades de educación ambiental sino de las personas que proponen espacios y colaboración para que los enseñantes construyan autónomamente proyectos de educación ambiental. Tanto en este caso, como también para la investigación ENSI, trabajar con la investigación-acción ha permitido no tanto evaluar los «productos» de los distintos laboratorios como explorar los «procesos», poner en evidencia los obstáculos, confrontar, con «agilidad» los valores escondidos y las epistemologías no explícitas (Magyar y Mayer, 1997).

Educación ambiental e investigación educativa coherente parecen, al menos desde un punto de vista de principios, indisolublemente ligadas. Es importante, por tanto, al extenderse las iniciativas de educación ambiental, al aumentar el número y el tipo de enseñantes y de operadores involucrados, que se extienda también la reflexión y la investigación sobre el sentido profundo de lo que se está haciendo. La educación ambiental, en efecto, puede ser un verdadero «caballo de Troya» para comprometer a todos los que se ocupan de educación en una reflexión sobre cuáles deben ser las nuevas modalidades de una educación para el futuro: una educación en la cual la utopía del progreso ceda el paso a la utopía de la sostenibilidad, en la que delegar al experto se transforme en asunción de responsabilidad, en la que las necesidades de globalización vayan de acuerdo con las exigencias y las diversidades locales.

\section{NOTA}

${ }^{1}$ Los más conocidos y utilizables para una correcta divulgación del debate actual sobre la evolución biológica y humana, son El pulgar del panda, La sonrisa de la libélula y La vida maravillosa.

* Ponencia presentada en el V Congreso Internacional sobre Investigación en la Didáctica de las Ciencias (Murcia, 10 a 13 de septiembre de 1997). 


\section{REFERENCIAS BIBLIOGRÁFICAS}

AMMASSARI, R. y PALLESCHI, M.T. (eds.) (1991). Educazione Ambientale: gli Indicatori di qualità, Isfol SR. Milán: Franco Angeli.

AUSUBEL, D. P. et al. (1983). Psicología educativa. Un punto de vista cognoscitivo. México: Trillas.

BACHELARD, G. (1983). La formación del espíritu científico. Buenos Aires: Siglo XXI.

BATESON, G. (1991). Pasos hacia una ecología de la mente. Buenos Aires: Planeta.

BATESON, G. (1982). Espíritu y Naturaleza. Buenos Aires: Amorrortu.

BRIANO, R. (1996). Due battute in rete: idee sul sistema nazionale per l'educazione ambientale. TD-Tecnologie Didattiche, Vol. 8-9, pp. 27-42.

BRIANO, R., MIDORO, V. y TRENTIN, G. (1996). Aggiornamento in rete dei docenti sull'Educazione Ambientale. TD-Tecnologie Didattiche, Vol. 8-9, pp.60-73.

BORGARELLO, G. y ZOBEL, B. (1996). Educazione e sviluppo sostenibile. Formazione Ambiente. Legambiente, ottobre dicembre, pp. 5-8.

CINI, M. (1994). Un Paradiso Perduto. Milán: Feltrinell.

CONTI, L. (1989). Prevedere l'imprevedibile, en Mayer, M. (ed.), Una scuola per l'ambiente. Risultati di una ricerca promossa dall'OCSE. I Quaderni di Villa Falconieri, Vol. 18 , Frascati, CEDE.

ELLIOTT, J. (1991). Environmental Education in Europe: Innovation, Marginalisation or Assimilation, en Environment, schools, and active learning. París: OECD-CERI.

ELLIOTT, J. (ed.). (1993). Recostructing Teacher Education. Londres-Washington DC: The Falmer Press.

ELLIOTT, J.(1995). Environmental education, action research and te role of the school. París: OECD: Environmental Learning for the 21 st Century.

FICHERA, A.M., MARCHEGGIANO, G., MAZZA, G. (1996). ANDREA: un servizio per operatori educativi. TD-Tecnologie Didattiche, Vol. 8-9, pp. 52-59.

FUL (1997). Education relative à l'environnement. Approches héorique et pratiques évaluatives. Environnememnt \& Société, Vol. 19.

VON FOERSTER, H. (1987). Sistemi che Osservano. Roma: Astrolabio.

GIL PÉREZ, D., CARRASCOSA, J., FURIÓ, C. y MARTÍNEZTORREGROSA, J. (1991). La enseñanza de las ciencias en la educación secundaria. Barcelona: ICE Horsori.

GINZBURG, C. (1986). Miti, emblemi, spie. Einaudi: Turín.

GIOLITTO, P. (ed.) (1997). L'Education à l'environnement dans l'Union Européenne. Luxembourg: Office de Publications Officielles des Communautés Européennes.

GLOBAL FORUM DE RÍO (1993). La Carta della Terra. Il manifesto dell' ambientalismo planetario. Turín: ISEDI, UTET.

GOULD, S. J. (1985). Il sorriso delfenicottero. Milán: Feltrinelli.

GOULD, S.J. (1991). La vida maravillosa. Barcelona: Crítica.

GOULD, S. J. (1994). Otto piccoli porcellini. Milán: Bompiani.
GUBA,E. y LINCOLN, Y.(1989). Fourth Generation Evaluation. Newbury Park: Sage.

LORENZONI, F. (1994). L'ospite bambino. Roma-Nápoles: Theoria.

LOSITO, B. y MAYER, M. (1995). Educazione Ambientale: una sfida per l'innovazione. Rapporto nazionale ENSI. Frascati: CEDE.

LOSITO, B. (1996). Valutare per capire: la valutazione a sostegno dei progetti di innovazione. TD-Tecnologie Didattiche, Vol. 8-9, pp. 43-51.

LUCAS, A.M. (1980-81). The role of science education in education for the environment. Journal of Environmental Education, Vol. 12(2), pp. 32-37.

MACDONALD, B. (1974). Evaluation and the control of Education en MacDonald, B.y Walker, R. (eds.), Evaluation, Research and the Problem of Control (SAFARI). Norwich: Centre for Applied Research in Education.

MAGYAR, A. y MAYER, M. (1997). Multi-level negotiations in constructing an action research: a Case Study on the 'explicit and implicit negotiations between Facilitators and Practitioners, Presentato al Simposio MOHD. Chicago: Annual meeting. Presso l'AERA (American Educational Research Association).

MATURANA, H. y VARELA, F. (1990). El árbol del conocimiento. Madrid: Debate.

MAYER, M. (1990). Conoscenza scientifica e conoscenza di senso comune. I Quaderni di Villa Falconieri, Vol. 22. Frascati: CEDE.

MAYER, M. (1994a). Complejidad y cambio: un enfoque dinámico de la educación ambiental. (Colec. Monografías del Máster en Educación Ambiental). Madrid: Fundación Universidad-Empresa.

MAYER, M. (1994b). Evaluating the outcomes of Environment and School Initiatives, en Evaluating Innovation in Environmental Education. París: OECD Documents.

MAYER, M. (1995). Quality indicators and innovation in environmental education. Environmental Learning for the 21st Century. París: OECD.

MAYER, M. (1996). L'Educazione Ambientale in Italia e in Europa, en Educazione Ambientale nelle scuole secondarie di $2^{\circ}$ grado in Umbria: esperienze a confronto. Perugia: Grella A. Ed., I Quaderni dell'IRRSAE Umbria, Vol. 9.

MAYER, M., MAGYAR, A, SETTI, F., ABELE, L. (1996). Training new educational professionals through Action Research. Rapporto presentato alla conferenza finale del Progetto MOHD, CEDE - Frascati, 4-7. Setiembre de 1996.

MAYER, M. (1997). Action research and the production of knowledge: the experience of an international project on environmental education, en Hollingsworth, S. (ed.). International Action research. A casebook for educational reform. Londres-Washington: Falmer Press.

MENTER, I. (1996). Action Research and School Initiatives in Environmental Education: a response to John Elliott. Environmental Education Research, Vol. 2(3), pp. 375-377.

MORIN, E. (1994). Introducción al pensamiento complejo. Barcelona: Gedisa.

NOVAK, J. y GOWIN, D.B. (1988). Aprendiendo a aprender. Barcelona: Martínez Roca. 
NOVO, M. (1995). La educación ambiental. Madrid: Universitas.

OECD (1995). Environmental Learning for the 21 st Century, París.

PARDO DÍAZ, A. (1995). La educación ambiental como proyecto. Barcelona: ICE-Horsori.

PATRIARCA, D. (1995). Verso un sistema nazionale per l'educazione ambientale. Formazione Ambiente. Octubrediciembre.

POSCH, P. (1991). Environment and school initiatives: background and basic premises of the project, en Environment, schools, and active learning. París: OECD-CERI.

POSCH P. (1993). Approaches to values in E.E., en Values in Environmental Education. Escocia: OECD-ECSI Project, Conference Report, Stirling.

POSCH, P. (1995). Professional development in environmental education: networking and infrastructures, en Environmental Learning for the 21st Century. París: OECD, p.110.

POZARNIC, B., SKERBINEK, M., MURKO, S. MLINAR, J. (1993). Torn between action and reflection: some dilemmas of pedagogical support persons in the Environment and School Initiatives Project. Educational Action Research, Vol. 1(3), pp. 493-509.

RAVETZ, J. y FUNTOVICZ, R. (1989). Usable knowledge, usable ignorance. A discourse on two sorts of science, en International Conference «The experts are categorical: Scientific Controversies and Political Decisions Concerning the Environment». París.

RAVETZ, J. (1992). Connaissance utile, ignorance utile?, en La Terre outragé: Les experts son formels! París: Editions Autrement, Série Sciences en Société.
ROBOTTOM, I. (1988). Social Critique or Social Control: some problems for Evaluation in Environmental Education. Journal of Research in Science Teaching, Vol. 26(5), pp. 435-443.

SAUVÉ, L. (1993) Education relative à l'environnement: representations et modes d'intervention, en L'education relative à l'environnement: pour un debat institutionel et méthodologique. Environnement et Société, Vol. 11, pp. 5-10.

STAKE, R.E.(1988). La valutazione 'responsive', en Giovannini, M.L. (ed.), La valutazione delle innovazioni nella scuola. Bolonia: Cappelli.

STENGERS, I. (1988). Complessità. Effetto di moda o problema?, en Stengers, (ed.), Da una scienza all'altra. Concetti nomadi. Florencia: Hopeful Monster.

STENGERS, I. (1992). Progres et complexité: tension entre deux images. Conferenza Internazionale Immagini della Società, della natura e della scienza attraverso l'educazione ambientale. Perugia: Italia.

STENHOUSE, L. (1984). Investigación y desarollo del curriculum. Madrid: Morata.

UNESCO (1992). Refonte de l'éducation pour un devéloppement durable. París: Dossiers Environnement et Développement.

OULTON, C. (1995). Rapporto sulla conferenza di Tolosa. Environmental Education Research, Vol. 1(2) (Unione Europea, DGXI, 1994).

WATZLAWICK, P., WEAKLAND, R. y FISCH, J. (1974). Change. Roma: Astrolabio. 